\title{
Relay Selection in Dual-Hop Vehicular Networks
}

\author{
Mehdi Seyfi, Student Member, IEEE, Sami Muhaidat, Member, IEEE, Jie Liang, Member, IEEE, and \\ Murat Uysal, Senior Member, IEEE
}

\begin{abstract}
In this letter, we investigate cooperative diversity with relay selection over cascaded Rayleigh fading channels. In particular, we analyze the performance of a relay selection scheme for cooperative vehicular networks with the decode-and-forward (DF) protocol. Only the "best" relay, which satisfies an index of merit, is selected. We ignore the direct transmission between the source $(S)$ and its destination $(D)$, and assume that the destination has perfect knowledge of the $S \rightarrow R$ and $R \rightarrow D$ channel gains. We study the performance of the underlying scheme in terms of outage probability and investigate its achievable diversity order.
\end{abstract}

Index Terms-Cooperative diversity, inter-vehicular networks, outage probability, relay selection.

\section{INTRODUCTION}

V EHICULAR ad-hoc networks (VANETs) [1] are a crucial component of the intelligent transportation systems which involve the application of advanced information processing, communications, sensor, and control technologies in an integrated manner to improve the functioning of the transportation system. VANETs have received much attention in recent years with their potential of enormous improvements in the road safety, and elimination of the excessive cost of traffic collisions. Besides safety improvement and traffic management, other applications, such as audio/video streaming, real-time gaming and high-speed internet access are also envisioned.

Considering the lack of infrastructure, cooperative diversity [2] has been recently proposed as an efficient solution to many challenging physical-layer problems in VANETs [3]. User cooperation takes advantage of the broadcast nature of the radiofrequency transmission and creates virtual antennas among the nodes which are willing to share their resources. Dual-hop transmission is a special case of user cooperation where no direct transmission between source and destination is possible. In this letter, we consider a dual-hop inter-vehicular communication system where a source vehicle communicates with a destination vehicle via another vehicle acting as a relay node. We assume

\footnotetext{
Manuscript received October 08, 2010; accepted December 13, 2010. Date of current version December 30, 2010. The work of J. Liang was supported in part by the Natural Sciences and Engineering Research Council (NSERC) of Canada under grants RGPIN312262-05, EQPEQ330976-2006, and STPGP350416-07. The associate editor coordinating the review of this manuscript and approving it for publication was Dr. Ioannis Psaromiligkos.

M. Seyfi, S. Muhaidat, and J. Liang are with the School of Engineering Science, Simon Fraser University, Burnaby, BC V5A 1S6 Canada (e-mail: mehdi_seyfi@sfu.ca; muhaidat@ieee.org; jiel@ sfu.ca).

M. Uysal is with the Faculty of Engineering, Özyeğin University, 34662 Istanbul, Turkey (e-mail: murat.uysal@ ozyegin.edu.tr).

Color versions of one or more of the figures in this paper are available online at http://ieeexplore.ieee.org.

Digital Object Identifier 10.1109/LSP.2010.2102017
}

that a number of vehicle nodes are willing to act as relays, therefore relay selection is performed among them.

Relay selection has been studied extensively in the literature, see, e.g., [6]-[10] and the references therein. However, current results are limited to Rayleigh fading channel assumption. Experimental and theoretical studies [4], [5] have reported cascaded (double) Rayleigh fading as an appropriate small-scale fading model for inter-vehicular channels. In this letter, we will investigate the outage performance and the achievable diversity order of dual-hop transmissions with relay selection over cascaded Rayleigh fading channels.

\section{SYSTEM MODEL}

We consider a multi-relay scenario with $M$ relays. We assume that the relay, $R_{m}, m=1, \ldots, M$, the source and destination terminals are equipped with single transmit and receive antennas. In our system model, $h_{s m}$ and $h_{m d}$ represent the channel coefficients between $S \rightarrow R_{m}$ and $R_{m} \rightarrow D$, respectively. Both hops are modeled as the product of two independent complex Gaussian random variables [11], $h_{s m} \triangleq h_{1_{m}} h_{2_{m}}$ and $h_{m d} \triangleq h_{3_{m}} h_{4_{m}}$, where $h_{k_{m}}$ is a complex Gaussian random variable with zero mean and variance of $\sigma_{k_{m}}^{2}$, for $k=1,2, \ldots, 4$ and $m=1,2, \ldots, M$. Therefore, $\left|h_{s m}\right|$ and $\left|h_{m d}\right|$ follow a cascaded Rayleigh distribution. We assume that all underlying channels are quasi-static which can be justified for vehicular communication scenarios in rush-hour traffic. We further assume, without loss of generality, that the additive white Gaussian noise (AWGN) terms have zero mean and variance of $N_{0}$. Assuming a half duplex constraint, the data transmission is performed in two time slots. In the first time slot the source terminal $S$ transmits its data to all the potentially available $M$ relays. Meanwhile, receiving the source signal via different independent channels, all the relays $R_{m}, m=1,2, \ldots M$, decode their received signal, and check whether the transmitted signal is decoded correctly or not. This can be done via some ideal CRC codes [10], which are added to the transmitted information symbols. We define the decoding set $\mathcal{D}(s)$ as the set of relays that decode the transmitted signal correctly. Clearly only those relay nodes with a minimal good source to relay channel can be in the decoding set $\mathcal{D}(s)$. In the second time slot, the best relay that satisfies an index of merit participates in the transmission and broadcasts its decoded symbol towards the destination. We assume that the source has a power constraint of $P$ Joules/symbol and similarly each relay node in $\mathcal{D}(s)$ can potentially transmit its information with $P$ Joules/symbol.

\section{Outage Probability}

Assuming that the communication between the source and the destination targets an end-to-end data rate $R$, the relay $R_{m}$ 
is in the decoding set $\mathcal{D}(s)$ if the $S \rightarrow R_{m}$ link observes an instantaneous capacity per bandwidth $C_{s m}$, that is above the required rate $R$

$$
C_{s m}=\frac{1}{2} \log _{2}\left(1+\alpha_{m}\right) \geq R
$$

where the factor $1 / 2$ accounts for the two time slot transmission protocol, $\alpha_{m}=\left(P / N_{o}\right) \alpha_{1 m} \alpha_{2 m}$, where $\alpha_{k m} \triangleq\left|h_{k_{m}}\right|^{2}$ for $k=1, \ldots 4$, and $m=1,2, \ldots, M$.

Proposition: The probability that the relay $R_{m}$ decodes the transmitted signal incorrectly is given by (2), where SNR = $P / N_{o}$ is the average signal to noise ratio (SNR) at the receiving node (see (2), shown at the bottom of the page).

Proof: Noting that $\alpha_{k m} \sim \lambda_{k_{m}} \exp \left(-\lambda_{k_{m}} \alpha_{k m}\right)$, for $k=$ $1, \ldots, 4$ and $m=1,2, \ldots, M$, is Rayleigh fading with $\lambda_{k_{m}}=$ $1 / E\left\{\alpha_{k m}\right\}$; hence, we can calculate the $\operatorname{CDF} F_{\alpha_{m}}(x)$ as

$$
\begin{aligned}
F_{\alpha_{m}}(x) & =\operatorname{Pr}\left(\alpha_{m} \leq x\right)=\operatorname{Pr}\left(\alpha_{1 m} \alpha_{2 m} \leq \frac{x}{\mathrm{SNR}}\right) \\
& =\int_{0}^{\infty} \operatorname{Pr}\left(\alpha_{1 m} \leq \frac{x}{\operatorname{SNR} \alpha_{2 m}} \mid \alpha_{2 m}\right) f_{\alpha_{2 m}}\left(\alpha_{2 m}\right) d \alpha_{2 m} \\
& =\int_{0}^{\infty} F_{\alpha_{1 m}}\left(\frac{x}{\operatorname{SNR} \alpha_{2 m}}\right) f_{\alpha_{2 m}}\left(\alpha_{2 m}\right) d \alpha_{2 m} \\
& =1-\lambda_{2 m} \int_{0}^{\infty} \exp \left(-\frac{\lambda_{1 m} x}{\operatorname{SNR} \alpha_{2 m}}\right) \exp \left(-\lambda_{2 m} \alpha_{2 m}\right) d \alpha_{2 m} \\
& =1-\sqrt{\frac{4 \lambda_{1 m} \lambda_{2 m} x}{\operatorname{SNR}}} K_{1}\left(\sqrt{\frac{4 \lambda_{1 m} \lambda_{2 m} x}{\operatorname{SNR}}}\right)
\end{aligned}
$$

where $K_{1}(\cdot)$ is the modified Bessel function of the second kind, and we have used the fact that [13]

$$
\int_{0}^{\infty} \exp \left(-\frac{a}{4 x}-b x\right) d x=\sqrt{\frac{a}{b}} K_{1}(\sqrt{a b}) .
$$

The outage probability of $S \rightarrow R_{m}$ link is subsequently obtained as

$$
\begin{aligned}
\operatorname{Pr}\left(C_{s m} \leq R\right) & =\operatorname{Pr}\left(\frac{1}{2} \log _{2}\left(1+\alpha_{m}\right) \leq R\right) \\
& =F_{\alpha_{m}}\left(2^{2 R}-1\right)
\end{aligned}
$$

Using (3) at $x=2^{2 R}-1$, we obtain (2).

Similarly, noting that $\beta_{m}=\left(P / N_{o}\right) \alpha_{3 m} \alpha_{4 m}, F_{\beta_{m}}(x)$ can be written as

$$
F_{\beta_{m}}(x)=1-\sqrt{\frac{4 \lambda_{3 m} \lambda_{4 m} x}{\mathrm{SNR}}} K_{1}\left(\sqrt{\frac{4 \lambda_{3 m} \lambda_{4 m} x}{\mathrm{SNR}}}\right) .
$$

In order to find the outage probability, following [12], we define a random variable $\gamma_{m}$ which represents the instantaneous SNR received at the destination via the $m$ th path. Typically, this random variable accounts for the channel gains of both $S \rightarrow$ $R_{m}$ and $R_{m} \rightarrow D$ links. Obviously, the $m$ th path would be in outage if any of the sub-channels, $S \rightarrow R_{m}$ or $R_{m} \rightarrow D$, is in outage. If the $S \rightarrow R_{m}$ is in outage, then the relay $R_{m}$ is not able to decode its received information. Therefore, no relaying would take place, i.e., the relay $R_{m}$ is not in the decoding set $\mathcal{D}(s)$, and the received SNR via the $m$ th path at the destination would be 0 . On the other hand, if the relay $R_{m}$ is on, i.e., it belongs to the decoding set $\mathcal{D}(s)$, the received SNR depends on the $R_{m} \rightarrow D$ link. In the following, we derive the probability density function (PDF) of the received SNR via the $m$ th path. First, the PDF of the received SNR given $R_{m}$ is off is

$$
f_{\gamma_{m} \mid R_{m} \text { is off }}(x)=\delta(x)
$$

where $\delta(x)$ is the Dirac delta function, and the probability of the event that $R_{m}$ is off is $\operatorname{Pr}\left(C_{s m} \leq R\right)$. On the other hand, the conditional PDF of the received SNR given $R_{m}$ is on is expressed by

$$
f_{\gamma_{m} \mid R_{m} \text { is on }}(x)=f_{\beta_{m}}(x)
$$

and this event is occurred with the probability 1 $\operatorname{Pr}\left(C_{s m} \leq R\right)$. Finally, the PDF of the received SNR $\gamma_{m}$, via the $m$ th path is obtained as [12]

$$
f_{\gamma_{m}}(x)=\operatorname{Pr}\left(C_{s m} \leq R\right) \delta(x)+\left[1-\operatorname{Pr}\left(C_{s m} \leq R\right)\right] f_{\beta_{m}}(x)
$$

and the cumulative density function (CDF), $F_{\gamma_{m}}(x)$, is obtained by integrating (8) with respect to $x$

$$
F_{\gamma_{m}}(x)=\operatorname{Pr}\left(C_{s m} \leq R\right)+\left[1-\operatorname{Pr}\left(C_{s m} \leq R\right)\right] F_{\beta_{m}}(x)
$$

where $F_{\beta_{m}}(x)$ is given in (5), and subsequently, $F_{\gamma_{m}}(x)$ is given by

$$
\begin{aligned}
F_{\gamma_{m}}(x)= & -\frac{4 x}{\operatorname{SNR}} \sqrt{\prod_{k=1}^{4} \lambda_{k m}} \\
& \times K_{1}\left(\sqrt{\frac{4 \lambda_{1 m} \lambda_{2 m} x}{\mathrm{SNR}}}\right) \\
& \times K_{1}\left(\sqrt{\frac{4 \lambda_{3_{m} \lambda_{4_{m}} x}}{\mathrm{SNR}}}\right) .
\end{aligned}
$$

It must be noted that the total received SNR via the selected relay $R_{m^{\star}}$ at the destination terminal is

$$
\gamma_{t}=\max _{m \in \mathcal{D}(s)}\left\{\beta_{m}\right\}
$$

however, using the random variable $\gamma_{m}$, the total received SNR can be written as

$$
\gamma_{t}=\gamma_{m^{\star}}=\max _{m \in M}\left\{\gamma_{m}\right\} .
$$

Note that the expressions in (11) and (12) are equal; however, the expression in (12) is analytically more tractable. The outage

$$
\operatorname{Pr}\left(C_{s m} \leq R\right)=1-\sqrt{\frac{4 \lambda_{1_{m}} \lambda_{2_{m}}\left(2^{2 R}-1\right)}{\mathrm{SNR}}} K_{1}\left(\sqrt{\frac{4 \lambda_{1_{m}} \lambda_{2_{m}}\left(2^{2 R}-1\right)}{\mathrm{SNR}}}\right) .
$$


probability of selection cooperation in the DF mode for intervehicular scenarios is then obtained as

$$
\begin{aligned}
\operatorname{Pr}\left(C_{s m^{\star}} \leq R\right) & =\operatorname{Pr}\left(\max _{m \in M}\left\{\gamma_{m}\right\} \leq 2^{2 R}-1\right) \\
& =\prod_{m=1}^{M} \operatorname{Pr}\left(\gamma_{m} \leq 2^{2 R}-1\right) \\
& =\prod_{m=1}^{M} F_{\gamma_{m}}\left(2^{2 R}-1\right) .
\end{aligned}
$$

Finally the closed-form outage probability formula is given by (14), shown at the bottom of the page.

\section{MAXIMUM ACHIEVABle Diversity ORDER}

In this section, we analyze the achievable diversity order for an inter-vehicular relay scenario with decode-and-forward selection cooperation, which to the best of our knowledge, has not been studied yet. We show that the maximum achievable diversity order for inter-vehicular relay channels with selection cooperation is $M-M \log (g(\mathrm{SNR}))$, where $M$ is the number of relays, and $g(\cdot)$ is a logarithmic function of SNR.

To simplify the analysis, without loss of generality, we assume $\lambda_{k m}=1$, for $k=1, \ldots, 4, m=1, \ldots, M$, and $\alpha_{k m}$ 's are i.i.d exponential random variables for $k=1,2$, and $m=$ $1, \ldots, M$. Considering the i.i.d property of $\alpha_{m}$ 's and $\beta_{m}$ 's we have $\operatorname{Pr}\left(C_{s m} \leq R\right)=F_{\alpha_{m}}\left(R_{o}\right)=F_{\beta_{m}}\left(R_{o}\right)$ where $R_{o}=$ $2^{2 R}-1$. By (9) we have

$$
\begin{aligned}
F_{\gamma_{m}}\left(R_{o}\right) & =2 F_{\beta_{m}}\left(R_{o}\right)-F_{\beta_{m}}^{2}\left(R_{o}\right) \\
& \leq 2 F_{\beta_{m}}\left(R_{o}\right) \\
& \simeq-\frac{8 R_{o}}{\operatorname{SNR}} \log \left(\sqrt{\frac{R_{o}}{\operatorname{SNR}}}\right)
\end{aligned}
$$

where we approximate $F_{\beta_{m}}\left(R_{o}\right)=1-\sqrt{\left(4 R_{o} / \mathrm{SNR}\right)} K_{1}$ $\left(\sqrt{4 R_{o} / \mathrm{SNR}}\right) \quad$ by $\quad-\left(4 R_{o} / \mathrm{SNR}\right) \log \left(\sqrt{R_{o} / \mathrm{SNR}}\right) \quad$ for $\left(R_{o} / \mathrm{SNR}\right) \ll 1$.

Finally, noting that $\gamma_{m}$ 's are i.i.d random variables and using (13) and (15), we obtain an upper bound on the outage probability of the underlying selection scheme as follows:

$$
\begin{aligned}
\operatorname{Pr}\left(C_{s m^{\star} d} \leq R\right) & =\left[F_{\gamma_{m}}\left(R_{o}\right)\right]^{M} \\
& \leq\left[-\frac{8 R_{o}}{\mathrm{SNR}} \log \left(\sqrt{\frac{R_{o}}{\mathrm{SNR}}}\right)\right]^{M} .
\end{aligned}
$$

The asymptotical diversity order $d$ is given by the magnitude of the slope of SER against average SNR in a log-log scale [4]:

$$
d=\lim _{\text {SNR } \rightarrow \infty}-\frac{\log \left(\operatorname{Pr}\left(C_{s m^{\star} d} \leq R\right)\right)}{\log \operatorname{SNR}}
$$

hence, using (16), we can write the asymptotical diversity order of the underlying selection scheme as

$$
d \simeq \lim _{\operatorname{SNR} \rightarrow \infty} M-\frac{M \log \left(\log (\mathrm{SNR})-\log \left(R_{o}\right)\right)}{\log \operatorname{SNR}} .
$$

The second term in (17) can be shown to tend zero by a simple l'Hôpital's rule. Thus, the outage probability of selection cooperation in inter-vehicular scenarios scales no slower than $\mathcal{O}\left(\mathrm{SNR}^{-M}\right)$, and hence, the maximum achievable diversity order is $M$.

\section{Simulation Results}

We consider two different power modes, namely, fractional power allocation (FPA) and equal power allocation (PA) modes. In FPA, all participating nodes carry the same power, i.e., $P=$ $P_{o} / M+1$, where $P_{o}$ is the total power budget. On the other hand, in the PA scheme, the total available power is $P_{o}$ and once a relay is selected all the available power, $P_{o}$, is divided equally between the source terminal and the selected relay, i.e., $P=$ $P_{o} / 2$ each. Fig. 1 compares the outage probability of selection cooperation in the FPA mode with the full cooperation scheme, i.e., all the relays participate in communication. It can be easily deduced from this figure that as the number of relays increases, the outage probability decreases. Furthermore, it is noticed that relay selection in the FPA mode performs worse than the full cooperation scheme. Fig. 1 also compares the simulation results with the analytical results, which are obtained using (14). For all considered cases, the analytical expressions exactly match with the simulation results.

Fig. 2 shows the outage probability of selection cooperation in the DF mode assuming the PA scheme. It is clear from Fig. 2 that, selection cooperation outperforms the full cooperation scheme. It is also observed that, as the number of relays increase the gap between selection cooperation and full cooperation further increases. For the sake of fair comparison in both scenarios, we assume that, in the full cooperation scheme, the power of $P=P_{o} / M+1$ is allocated to each transmitting node.

In Fig. 3, the selection cooperation scheme in cascaded Rayleigh channels is compared with its counterpart in Rayleigh fading channels. As it is obvious from Fig. 3, selection cooperation in Rayleigh fading channels enjoys a better performance than cascaded Rayleigh channels.

Fig. 4 shows the diversity order analysis of selection cooperation in a cascaded Rayleigh fading channel versus its Rayleigh fading counterpart. As depicted in the figure as the SNR tends to infinity, the diversity order of both channels models approaches $M$, however, in a Rayleigh fading channel, the full diversity order is achieved in lower SNR values. This can also be seen in Fig. 3 where the slope of the Rayleigh fading channel curves

$$
\operatorname{Pr}\left(C_{s m^{\star} d} \leq R\right)=\prod_{m=1}^{M}\left[1-4 \frac{\left(2^{2 R}-1\right)}{\mathrm{SNR}} \sqrt{\prod_{k=1}^{4} \lambda_{k m}} K_{1}\left(\sqrt{4 \lambda_{1 m} \lambda_{2 m} \frac{\left(2^{2 R}-1\right)}{\mathrm{SNR}}}\right) K_{1}\left(\sqrt{4 \lambda_{3_{m}} \lambda_{4_{m}} \frac{\left(2^{2 R}-1\right)}{\mathrm{SNR}}}\right)\right] .
$$




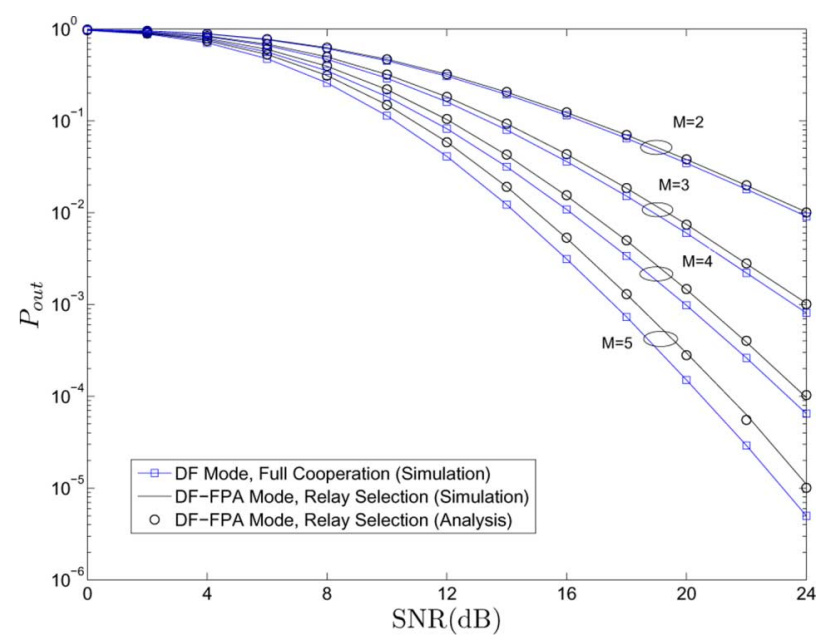

Fig. 1. Outage probability of DF selection and regular cooperation for equal power scheme, $P=P_{o} / M+1$ and $m=2, \ldots 5$.

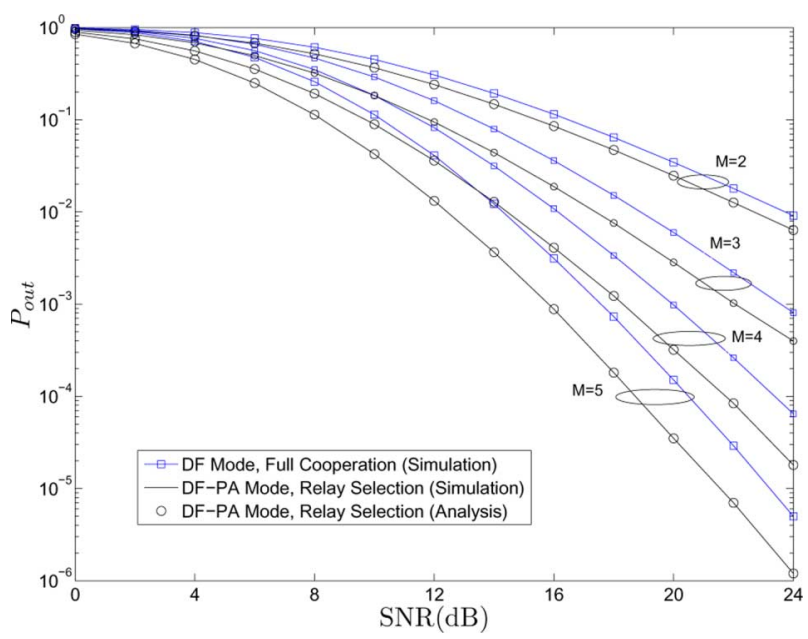

Fig. 2. Outage probability of DF selection and regular cooperation for power allocation scheme, $P=P_{o}$ and $m=2, \ldots 5$.

are slightly steeper than the corresponding cascaded Rayleigh channel curves at the same SNRs.

\section{CONCLUSION}

In this letter, we discuss selection cooperation in DF vehicular networks, where the channels in each link are modeled as cascaded Rayleigh fading. We derive an exact analytical expression for the outage probability and prove that the maximum achievable diversity order is equal to the number of relays.

\section{REFERENCES}

[1] H. Hartenstein and K. P. Laberteaux, "A tutorial survey on vehicular ad hoc networks," IEEE Commun. Mag., vol. 46, no. 6, pp. 164-171, Jun. 2008.

[2] M. Dohler and Y. Li, Cooperative Communications: Hardware, Channel and PHY. Hoboken, NJ: Wiley, 2010.

[3] H. Ilhan, M. Uysal, and I. Altunbas, "Cooperative diversity for intervehicular communication: Performance analysis and optimization," IEEE Trans. Veh. Technol., vol. 58, no. 7, pp. 3301-3310, Sep. 2009.

[4] R. U. Nabar, H. Bolcskei, and F. W. Kneubuhler, "Fading relay channels: Performance limits and space-time signal design," IEEE J. Sel. Areas Commun., vol. 22, no. 6, pp. 1099-1109, Aug. 2004.

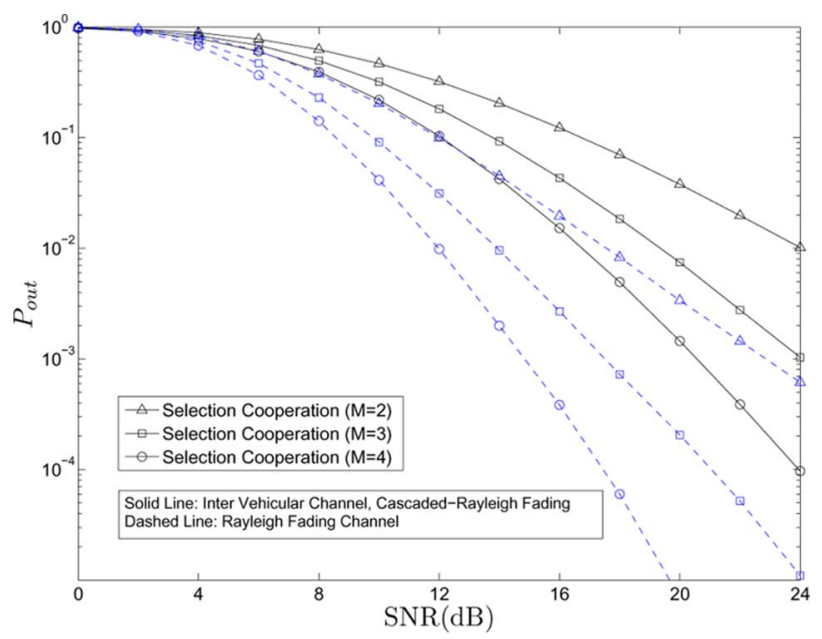

Fig. 3. Outage probability of DF selection cooperation in cascaded Rayleigh and Rayleigh fading channels for $m=2, \ldots 4$.

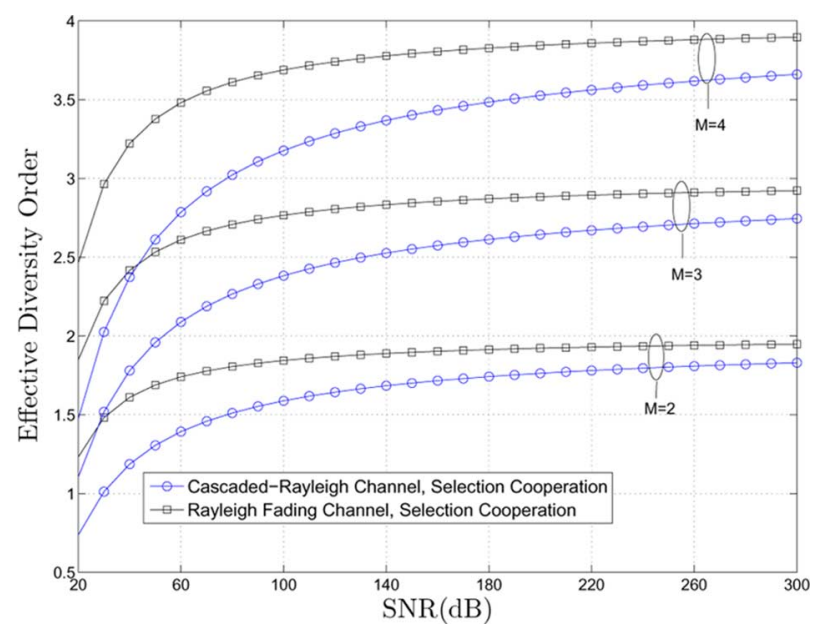

Fig. 4. Effective diversity order versus SNR for DF selection cooperation in cascaded Rayleigh channels for $m=2, \ldots 5$.

[5] V. Erceg et al., "Comparisons of a computer-based propagation prediction tool with experimental data collected in urban microcellular environments," IEEE J. Sel. Areas Commun., vol. 15, no. 4, pp. 677-684, May 1997.

[6] A. Bletsas, H. Shin, and M. Z. Win, "Outage-optimal cooperative communications with regenerative relays," in Proc. Conf. Inf. Sci. Syst. (CISS2006), Mar. 2006, pp. 623-637.

[7] D. S. Michalopoulos and G. K. Karagiannidis, "Performance analysis of single-relay selection in rayleigh fading," IEEE Trans. Wireless Commun., vol. 7, no. 10, pp. 3718-3724, Oct. 2008.

[8] S. Ikki and M. H. Ahmed, "Exact error probability an channel capacity of the best-relay cooperative-diversity networks," IEEE Signal Process. Lett., vol. 16, no. 12, Dec. 2009.

[9] S. Ikki and M. H. Ahmed, "Performance analysis of adaptive decodeand-forward cooperative diversity networks with the best relay selection," IEEE Trans. Commun., vol. 58, no. 1, pp. 68-72, Jan. 2010.

[10] E. Beres and R. Adve, "Selection cooperation in multi-source cooperative networks," IEEE Trans. Wireless Commun., vol. 7, no. 1, pp. 118-127, Jan. 2008.

[11] M. Uysal, "Maximum achievable diversity order for cascaded rayleigh fading channels," IEE Electron. Lett., vol. 41, no. 23, pp. 43-44, Nov. 2005.

[12] N. C. Beaulieu and J. Hu, "A closed-form expression for the outage probability of decode-and-forward relaying in dissimilar rayleigh fading channels," IEEE Commun. Lett., vol. 10, no. 12, pp. 813-815, Dec. 2006.

[13] I. S. Gradshteyn and I. M. Ryzhik, Table of Integrals, Series and Products, 7th ed. New York: Elsevier, 2007. 